BI-TP 2012/05

\title{
Thermal production of ultrarelativistic right-handed neutrinos: Complete leading-order results
}

\author{
Denis Besak, 1 Dietrich Bödeker 2 \\ Fakultät für Physik, Universität Bielefeld, D-33615 Bielefeld, Germany
}

\begin{abstract}
The thermal production of relativistic right-handed Majorana neutrinos is of importance for models of thermal leptogenesis in the early Universe. Right-handed neutrinos can be produced both by $1 \leftrightarrow 2$ decay or inverse decay and by $2 \rightarrow 2$ scattering processes. In a previous publication we have studied the production via $1 \leftrightarrow 2$ (inverse) decay processes. There we have shown that multiple scattering mediated by soft gauge boson exchange also contributes to the production rate at leading order and gives a strong enhancement. Here we complete the leading order calculation by adding $2 \rightarrow 2$ scattering processes involving either electroweak gauge bosons or thirdgeneration quarks. We find that processes with gauge interactions give the most important contributions. We also obtain a new sum rule for the Hard Thermal Loop resummed fermion propagator.
\end{abstract}

\footnotetext{
${ }^{1}$ denis.besak@gmx.de

${ }^{2}$ bodeker@physik.uni-bielefeld.de
} 


\section{Contents}

1 Introduction and motivation $\quad 1$

2 Production rate and processes 4

$3 \quad 1 \leftrightarrow 2$ scattering including soft gauge interactions 6

3.1 Kinematics . . . . . . . . . . . . . . . . . . 6

3.2 Computing the rate ..................... 8

$42 \rightarrow 2$ scattering $\quad 10$

4.1 Hard momentum transfer . . . . . . . . . . . . . . . . . 10

4.2 Soft momentum transfer . . . . . . . . . . . . . . . . 12

5 Numerical results $\quad[14$

6 Summary and Conclusions $\quad 18$

A Details of the calculation of the $2 \rightarrow 2$ rate $\quad 19$

A.1 s-channel parametrization ..................... 19

A.1.1 quark scattering . . . . . . . . . . . . . . 20

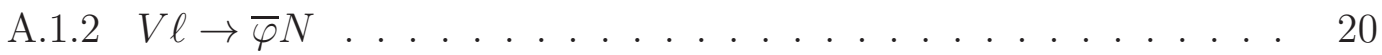

A.2 $t$-channel parametrization: hard contribution . . . . . . . . 20

A.2.1 $\ell \varphi \rightarrow V N \ldots \ldots \ldots \ldots \ldots \ldots$

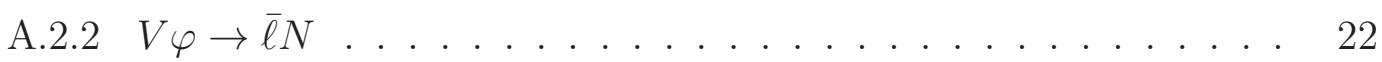

B Sum rule for HTL resummed fermion propagotor $\quad 22$

\section{Introduction and motivation}

One of the outstanding problems of standard cosmology is to explain the origin of the asymmetry between matter and antimatter. Without such an asymmetry, all the structures we observe today would have never formed and mankind would not exist. The asymmetry can be expressed as the baryon-to-photon ratio

$$
\frac{n_{B}}{n_{\gamma}}=(6.19 \pm 0.15) \cdot 10^{-10}
$$

whose numerical value is obtained from a combined analysis of data for large-scale structure and the spectrum of the Cosmic Microwave Background [1]. 
In order to obtain a net baryon asymmetry, only three conditions need to be met, as outlined by Sakharov in his seminal paper [2]. Yet, providing a model that can successfully explain the measured baryon-to-photon ratio remains a challenging task. Several different scenarios how to realize the Sakharov conditions have been devised [3]. In the last decade, leptogenesis [4] has become very popular. The basic idea of most leptogenesis models is to extend the Standard Model (SM) by adding heavy righthanded neutrinos $N_{\mathrm{R}}$ with Majorana mass $M_{N}$. In the simplest realization they interact with the SM particles via a Yukawa coupling to ordinary, left-handed leptons $\ell_{\mathrm{L}}$ and the Higgs bosons $\varphi$ as follows:

$$
\mathscr{L}_{\text {int }}=h_{i j} \overline{N_{\mathrm{R} i}} \widetilde{\varphi}^{\dagger} \ell_{\mathrm{L} j}+\text { h.c. } .
$$

Here $\widetilde{\varphi} \equiv i \sigma^{2} \varphi^{*}$ with the Pauli matrix $\sigma^{2}$ is the isospin conjugate of $\varphi$. Furthermore, the indices $i, j$ label the fermion families, and $h_{i j}$ is the Yukawa coupling matrix which need not be diagonal.

The Majorana neutrinos are unstable and decay both into leptons and antileptons, $N \rightarrow \ell \varphi, N \rightarrow \bar{\ell} \bar{\varphi}$. The $C P$ symmetry is violated and the corresponding decay rates are not equal. Therefore, an excess of antileptons over leptons can be generated. The resulting asymmetry is converted into an excess of baryons over antibaryons via the sphaleron transitions which conserve $B-L$ but violate $B+L$ [5]. In addition to providing a source for the measured baryon asymmetry, this scenario offers a framework to explain the smallness of the neutrino masses via the seesaw mechanism [6]. This twofold virtue is what makes the scenario of leptogenesis particularly appealing.

So far there is no complete leading order treatment of leptogenesis, for the case of relativistic Majorana neutrinos. It has been argued that there are theoretical uncertainties of a factor 2 or more [7]. The purpose of this paper is to make a step towards a complete leading order treatment. We compute the complete leading order production rate of Majorana neutrinos in the symmetric phase of the electroweak theory, 3 in the regime $T \gg M_{N}$. It is one ingredient in the set of kinetic equations which describe leptogenesis. At the end of inflation the number density of right-handed neutrinos should be negligible, and therefore the production rate determines the initial conditions for leptogenesis. Thus it is particularly important in the so-called weak washout regime, where the Majorana neutrinos are not close to equilibrium. It is essential when their number density always remains far below the equilibrium density. This is the case in scenarios where the asymmetry is generated via oscillations between the right-handed

\footnotetext{
${ }^{3}$ Results for the broken phase can be found in Ref. [8]. The production rate in the low temperature regime has recently been calculated at next-to-leading order 9] correcting an earlier calculation in Ref. [10].
} 
neutrinos [11, 12]. These types of models also can potentially explain both the baryon asymmetry and dark matter [12, 13].

Two types of processes contribute to the leading order production rate. The first includes the inverse decay $\varphi \ell \rightarrow N$. At high temperature all masses are smaller than the typical energies. Then the momenta in this process are nearly collinear. It is therefore quite sensitive to thermal mass effects [14, 15, 16]. The above process is kinematically forbidden at high temperature, and the decay $\varphi \rightarrow \ell N$ becomes possible. 4] In [16] it was shown that processes with additional scattering mediated by soft electroweak gauge bosons exchange also contribute at leading order. 5 The soft scattering opens new decay and inverse decay channels. One has to sum over arbitrary numbers of such interactions which was done in Ref. [16], where it turned out that including them increases the rate by almost a factor 3 .

However, the calculation in [16] is still incomplete: $2 \rightarrow 2$ scattering processes involving hard $(p \sim T)$ electroweak gauge bosons or third-generation quarks contribute to the leading order production rate as well. Quark-initiated processes have been taken into account by many authors (see e.g. [18, 19, 20, 21]), explicit results for the corresponding rate were shown in [19, 21]. Gauge boson scattering processes have been considered in Ref. [14, 19]. They contain a leading order contribution with the exchange of a soft fermion in the $t$-channel, which is infrared divergent when medium effects are not taken into account. So far no consistent computation, which properly treats the soft exchange contribution has been carried out. The goal of this publication is to close this gap and complete the treatment of the thermal production of Majorana neutrinos in a hot electroweak plasma.

This paper is organized as follows. In Sec. 2 we review the processes which can create a population of right-handed neutrinos, and present equations for the differential production rate. The results of Ref. [16] for the collinear emission processes including soft gauge boson exchange scattering are briefly reviewed in Sec. 3. Then in Sec. 4 we describe the calculation of the production rate due to $2 \rightarrow 2$ scattering processes off hard quarks or electroweak gauge bosons. Our main result, the total $2 \rightarrow 2$ rate, is shown in Eq. (29) of Sec. 5. This section also contains numerical results for the total and differential rates. In Sec. [6 we then conclude. Appendix A contains technical details of the calculation of the $2 \rightarrow 2$ scattering rate with hard momentum transfer.

\footnotetext{
${ }^{4}$ In [14] a lepton mass corresponding to soft fermionic excitations was used. However, at leading order the dominant contribution is due to hard leptons, and one has to use the so-called asymptotic mass instead [16. This was improved in Ref. [15] by resumming the Hard Thermal Loop fermion self-energy. For hard momenta this is equivalent, at leading order, to using the asymptotic mass.

${ }^{5}$ Among other contributions, they include a resummation of a finite width of the Standard Model particles, the role of which was recently emphasized in [17].
} 
In Appendix $\mathrm{B}$ we show that the Hard Thermal Loop resummed propagator satisfies a sum rule which greatly simplifies the calculation of the $2 \rightarrow 2$ rate with soft fermion exchange in Sec. 4 .

Conventions and notation The signature of the metric is +--- , 4-vectors are denoted by lower case italics, 3 -vectors by boldface italics.

\section{Production rate and processes}

We consider a hot electroweak plasma that is fully equilibrated, except for the righthanded Majorana neutrinos, which are assumed to have negligible number density. We compute their production rate $\Gamma$, i.e., the number of right-handed neutrinos which are thermally produced per unit time and unit volume, at leading order in the Yukawa interaction (2). We focus on the lightest Majorana neutrinos $N_{1} \equiv N$, which we assume to be the dominant source of lepton asymmetry. We include all contributions of leading order in the $\mathrm{SU}(2)$ and $\mathrm{U}(1)$ gauge couplings $g$ and $g^{\prime}$, the top quark Yukawa coupling constant $h_{t}$ and the Higgs self-coupling $\lambda$. For the power counting we assume that all these couplings are of the same order and collectively refer to them by $g$. All other SM couplings are neglected. We perform the computation in the high-temperature regime where $M_{N} \ll T$. This allows us to formally treat the mass of the Majorana neutrino as being soft, $M_{N} \sim g T$, and therefore parametrically of the same order as the thermal Higgs and lepton masses. The leading order rate is then of order $h^{2} g^{2}$ where

$$
h^{2} \equiv \sum_{j}\left|h_{1 j}\right|^{2}
$$

There are two types of processes by which right-handed neutrinos are produced at leading order. Among the first are the $1 \leftrightarrow 2$ processes (see Fig. 1) $(a)$ )

$$
\varphi \ell \rightarrow N, \quad \varphi \rightarrow \bar{\ell} N, \quad \ell \rightarrow \bar{\varphi} N
$$

At low temperature only the first one is kinematically allowed. When $T$ increases, the thermal masses $m_{\varphi}$ and $m_{\ell}$ (see Eqs. (10), (11) ) increase. On the other hand, the thermal mass of the right-handed neutrinos is negligible. Therefore $m_{\varphi}+m_{\ell}$ can become larger than $M_{N}$, so that the inverse $N$-decay is kinematically no longer allowed. At even higher temperature $m_{\varphi}>M_{N}+m_{\ell}$. Then the Higgs decay in (4) becomes possible. The third process in Eq. (4) is not allowed at any temperature because $m_{\varphi}>m_{\ell}$. However, including multiple interactions with soft gauge boson exchange as shown in Fig. 1(b) changes this picture. Then the decay/recombination processes do occur at any temperature and it also makes the process $\ell \rightarrow \bar{\varphi} N$ possible. As was demonstrated 


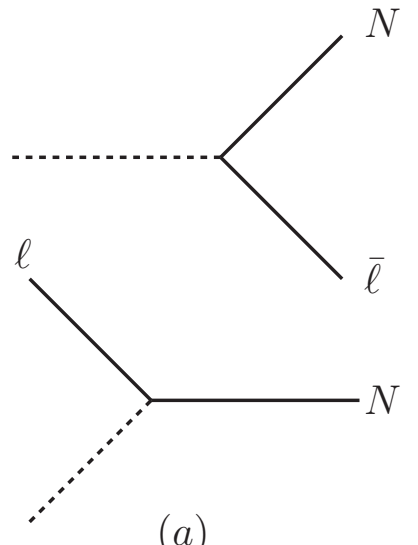

(a)

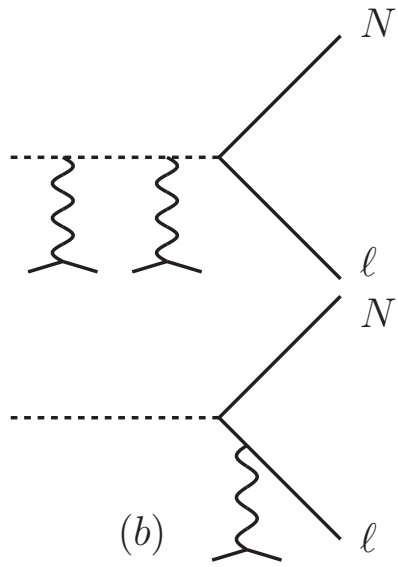

(b)

Figure 1: Leading order contributions to the production of right-handed neutrinos $N$. (a) Decay and recombination processes which occur when the asymptotic thermal masses are such that they are kinematically allowed. (b) Examples for processes, where exchanges of soft gauge bosons with particles in the plasma have been added. Higgs bosons are denoted with a dashed line and gauge bosons with a wiggled line. Fermions are represented by solid lines.

in [16], these processes with an arbitrary number of soft gauge interactions contribute at leading order.

The second type are the $2 \rightarrow 2$ hard particle scattering processes shown in Fig. 2 , They can also occur at any temperature. There are processes involving quarks,

$$
Q_{3} \bar{t} \rightarrow \ell N, \quad t \ell \rightarrow Q_{3} N, \quad \bar{Q}_{3} \ell \rightarrow \bar{t} N
$$

where $t$ denotes the right-handed top quark and $Q_{3}$ the doublet of left-handed thirdgeneration quarks. They contribute at the order $h^{2} h_{t}^{2}$ and thus need to be taken into account in a complete leading order computation. Additionally, there are processes involving $\mathrm{SU}(2)$ or $\mathrm{U}(1)$ gauge bosons $V$,

$$
\ell \varphi \rightarrow N V, \quad \ell V \rightarrow N \bar{\varphi}, \quad \bar{\varphi} V \rightarrow N \ell
$$

which contribute at order $h^{2} g^{2}$ or $h^{2} g^{\prime 2}$, which means that they are also part of the leading order production rate. With each of these processes, we also need to include their $C P$ conjugate, where every particle is replaced by its antiparticle. At leading order there is no $C P$ violation, and the rates are the same.

For thermal field theory calculations it is convenient to express the rate $\Gamma$ in terms of the self-energy of the right-handed neutrinos. One can define the self-energy $\Sigma$ as usual if one promotes the right-handed spinor $N_{\mathrm{R}}$ to a 4-component Majorana-spinor $N \equiv N_{\mathrm{R}}+N_{\mathrm{R}}^{c}$ where $c$ stands for charge conjugation. Then $\Gamma$ can be obtained from 

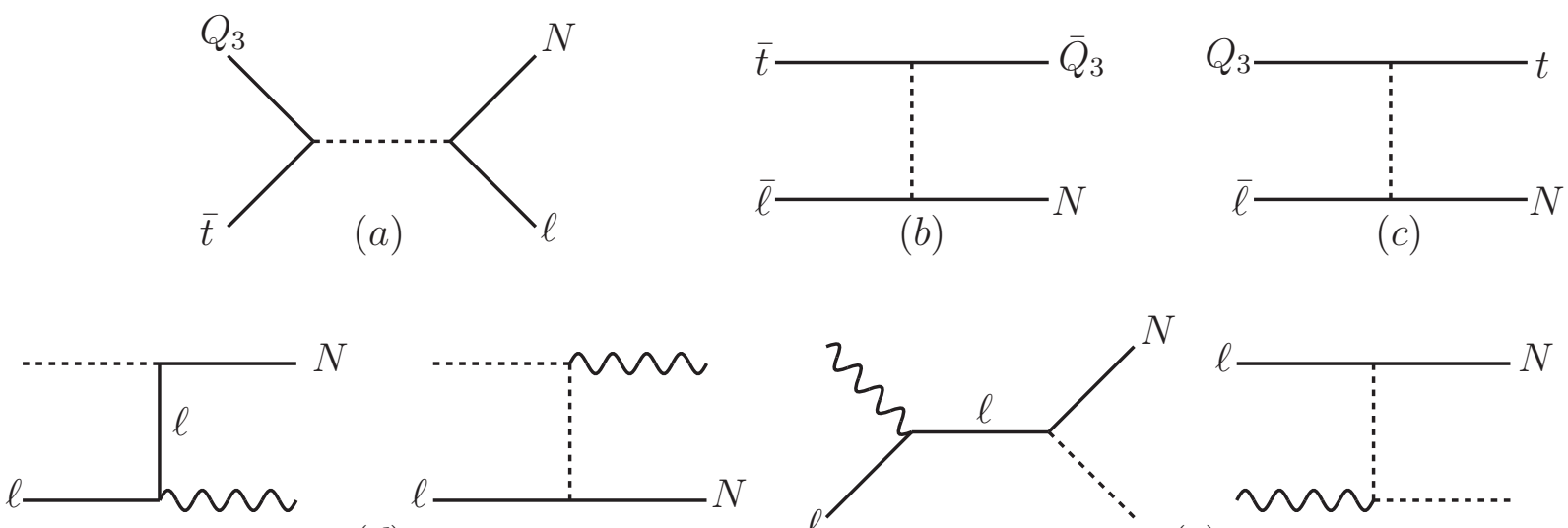

(d)
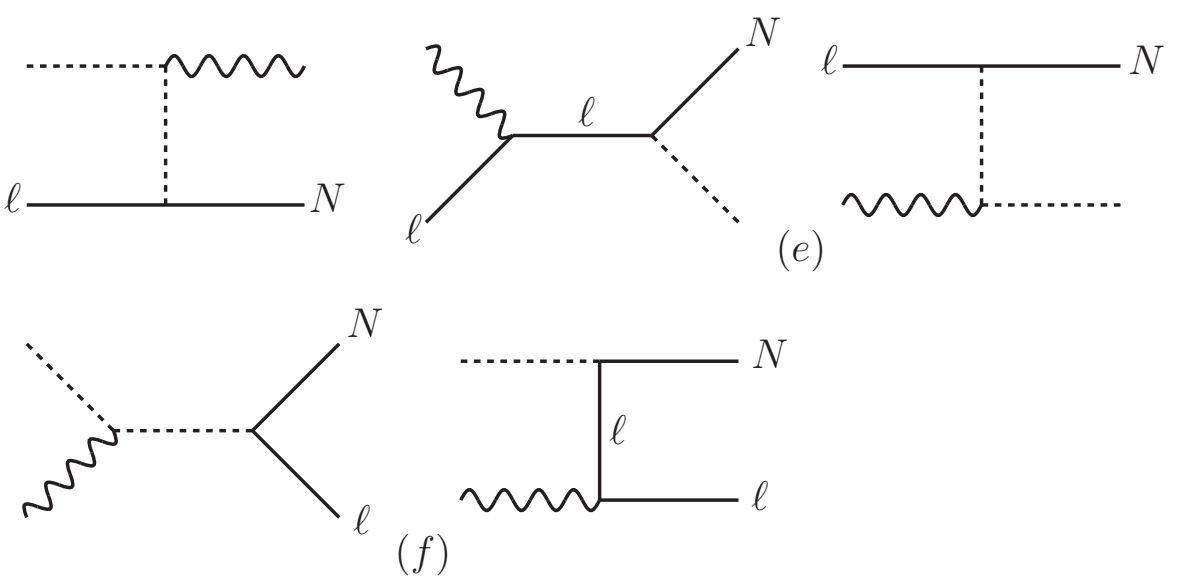

Figure 2: $2 \rightarrow 2$ scattering contributions to Majorana neutrino production.

the imaginary part of the retarded self-energy $\Sigma_{\text {ret }}(k)=\Sigma\left(k^{0}+i 0^{+}, \boldsymbol{k}\right)$, where $k^{0}$ and $\boldsymbol{k}$ are the (real) energy and the 3 -momentum of the produced neutrinos, through the relation [16]

$$
(2 \pi)^{3} 2 k^{0} \frac{d \Gamma}{d^{3} k}=2 f_{\mathrm{F}}\left(k^{0}\right) \operatorname{Im} \operatorname{Tr}\left[k \Sigma_{\text {ret }}(k)\right] .
$$

Here $f_{\mathrm{F}}$ is the Fermi-Dirac distribution function. Due to the Majorana-nature of $N$ there are two types of diagrams contributing to the self-energy which differ by the orientation of the internal lines. Both types of diagrams give the same contribution. We consider only one of them, and multiply by 2 to obtain the correct rate. This amounts to using

$$
(2 \pi)^{3} 2 k^{0} \frac{d \Gamma}{d^{3} k}=4 f_{\mathrm{F}}\left(k^{0}\right) \operatorname{Im} \operatorname{Tr}\left[k P_{\mathrm{L}} \Sigma_{\text {ret }}(k)\right]
$$

with the left-handed projector $P_{\mathrm{L}}=\left(1-\gamma_{5}\right) / 2$.

\section{$3 \quad 1 \leftrightarrow 2$ scattering including soft gauge interactions}

\subsection{Kinematics}

First consider the processes shown in Fig. 1. All external momenta in Fig. 1( $(a)$ are hard $(p \sim T)$. The mass of $N$ as well as the thermal masses are soft, i.e., of order $g T$. 
Therefore the typical angles between the hard momenta are of order $g$. We assume $g$ to be small, which implies that all momenta are nearly collinear. Fig. 1( $b)$ shows processes with additional interactions mediated by soft electroweak gauge bosons. With these soft interactions the momenta of $\varphi, \ell$ and $N$ are still nearly collinear. Furthermore, the hard 4-momenta are close to the light-cone, $p^{2} \sim g^{2} T^{2}$. We denote by $\boldsymbol{v} \equiv \boldsymbol{k} /|\boldsymbol{k}|$ the unit 3-vector $\boldsymbol{v}$ in the direction of the momentum $\boldsymbol{k}$ of the right-handed neutrino. For all vectors the components parallel to $\boldsymbol{v}$ are denoted by

$$
p_{\|} \equiv \boldsymbol{p} \cdot \boldsymbol{v}
$$

We further define the light-like vector $v \equiv(1, \boldsymbol{v})$. Then one has to account for three distinct momentum scales:

1. The emitting particles and the emitted particle have $p_{\|} \sim T$.

2. All 3-momenta perpendicular to $\boldsymbol{v}$ are soft, $\boldsymbol{p}_{\perp} \sim g T$. Also all components of the exchanged gauge boson momentum $q$ are soft, $q \sim g T$.

3. Finally, all 4-momenta $p$ satisfy $v \cdot p=\left(p_{0}-p_{\|}\right) \sim g^{2} T$.

For momenta with $p^{2} \sim g^{2} T^{2}$ one cannot neglect the modification of the dispersion relations which is caused by the interactions with the hot plasma. For hard particles the dispersion relation can be written as $p^{2}=m^{2}$, where $m$ is the so-called asymptotic mass [22]. 6] For the Higgs and the lepton doublets they are given by 7

$$
\begin{aligned}
& m_{\varphi}^{2}=\frac{1}{16}\left(3 g^{2}+4 y_{\ell}^{2} g^{2}+4 h_{t}^{2}+8 \lambda\right) T^{2}, \\
& m_{\ell}^{2}=\frac{1}{16}\left(3 g^{2}+4 y_{\ell}^{2} g^{2}\right) T^{2}
\end{aligned}
$$

where $g$ and $g^{\prime}$ are the $\mathrm{SU}(2)$ and $\mathrm{U}(1)$ gauge couplings, $y_{\ell}=-1 / 2$ is the weak lepton hypercharge, $h_{t}$ is the top Yukawa coupling and $\lambda$ is the Higgs self-coupling. Note that the gauge field contributions to the asymptotic masses for Higgs and leptons are equal (cf. Refs. [23, 24]). However, the Higgs also receives important contributions from the Yukawa interaction with the top quark and from the Higgs self-interaction, so that $m_{\varphi}>m_{\ell}$. All other contributions can be neglected due to the smallness of the

\footnotetext{
${ }^{6}$ It is oftentimes referred to as $m_{\infty}$.

${ }^{7}$ For scalars the asymptotic mass is the same as the thermal self-energy computed for vanishing momentum, which enters the finite temperature effective potential for the scalar field. It also equals the frequency of scalar field oscillations with zero $\boldsymbol{p}$. For fermions, however, the asymptotic mass is larger than the oscillation frequency for vanishing $\boldsymbol{p}$ by a factor $\sqrt{2}[22$.
} 


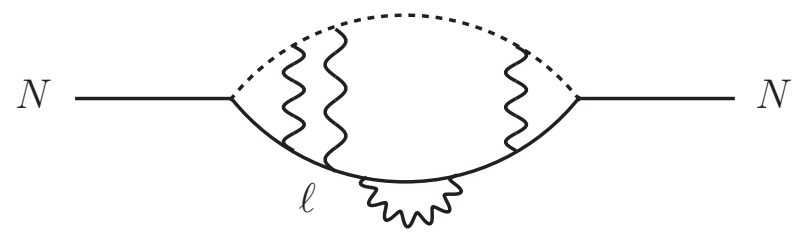

Figure 3: Example for a self-energy diagram that needs to be taken into account in a consistent leading order calculation of the production rate via Eq. (7).

corresponding coupling constants. Also, the thermal mass of the Majorana neutrinos can be neglected.

The relevance of thermal masses for these processes was first realized in Ref. [14]. There, however, the thermal mass for soft fermionic excitations was used, by which the rate is overestimated. In [15] it was argued that the lepton and/or the Higgs momenta are soft, and that it is therefore necessary to do a Hard Thermal Loop resummation for the Higgs and charged lepton lines. This is correct at the edge of the threshold where the decay becomes kinematically allowed. Since the Hard Thermal Loop gives the correct asymptotic mass even when the external momentum is hard, the result of Ref. [15] contains the correct decay contribution to the rate also away from the thresholds. However, the dominant contribution with this collinear kinematics is obtained by adding interactions with other hard particles in the plasma, mediated by the exchange of soft electroweak gauge bosons as shown in Fig. 1(b) [16]. In a complete leading order calculation an arbitrary number of such interactions has to be taken into account. We summarize the results that were already obtained in [16], to which we refer the reader for the derivation.

\subsection{Computing the rate}

As shown in [16], the kinematics described above necessitates the inclusion of multiple soft scattering already at leading order. Examples for processes that must be taken into account are shown in Fig. 1(b). In order to find the production rate due to this infinite set of processes, it is most convenient to use (7) with the self-energy given by diagrams of the form shown in Fig. 3 with an arbitrary number of soft gauge boson ladder rungs or self-energy insertions. The self-energy to be inserted in (17) is obtained by resumming all diagrams which respect the topology as given in Fig. 3: No crossed 
ladder rungs or nested loops due to self-energy insertions on the hard lines in the loop may occur, but the number of gauge bosons and thus the total number of loops is unlimited. This means that there is a clear mismatch between coupling constant expansion and loop expansion: Adding more loops does not suppress the resulting rate as long as we respect the kinematics outlined in Sec. 3.1.

Because of this complexity, it is not possible to give an explicit result for the retarded self-energy to be inserted in (17). Instead, the diagrammatic resummation leads to an integral equation that has to be solved numerically to obtain the rate. The retarded self-energy can be expressed by a 2-dimensional vector function $\boldsymbol{f}$ and a scalar function $\psi$. We refer the reader to [16] for details and only present the final result:

$$
(2 \pi)^{3} 2 k_{\|} \frac{d \Gamma^{1 \leftrightarrow 2}}{d^{3} k}=-d(r) h^{2} \int \frac{d^{3} p}{(2 \pi)^{3}} \frac{f_{\mathrm{F}}\left(p_{\|}\right) f_{\mathrm{B}}\left(k_{\|}-p_{\|}\right)}{k_{\|}-p_{\|}} \operatorname{Re}\left[\frac{k_{\|}}{2 p_{\|}^{2}} \boldsymbol{p}_{\perp} \cdot \boldsymbol{f}+\frac{M_{N}^{2}}{k_{\|}} \psi\right]
$$

where $d(r)=2$ is the dimension of the gauge group representation and $\boldsymbol{f}$ and $\psi$ obey the integral equations

$$
\begin{aligned}
& i \epsilon(k, \boldsymbol{p}) \boldsymbol{f}\left(\boldsymbol{p}_{\perp}\right)-\int \frac{d^{2} q_{\perp}}{(2 \pi)^{2}} \mathscr{C}\left(\boldsymbol{q}_{\perp}\right)\left[\boldsymbol{f}\left(\boldsymbol{p}_{\perp}\right)-\boldsymbol{f}\left(\boldsymbol{p}_{\perp}-\boldsymbol{q}_{\perp}\right)\right]=2 \boldsymbol{p}_{\perp}, \\
& i \epsilon(k, \boldsymbol{p}) \psi\left(\boldsymbol{p}_{\perp}\right)-\int \frac{d^{2} q_{\perp}}{(2 \pi)^{2}} \mathscr{C}\left(\boldsymbol{q}_{\perp}\right)\left[\psi\left(\boldsymbol{p}_{\perp}\right)-\psi\left(\boldsymbol{p}_{\perp}-\boldsymbol{q}_{\perp}\right)\right]=1
\end{aligned}
$$

with the kernel

$$
\mathscr{C}\left(\boldsymbol{q}_{\perp}\right) \equiv T\left[C_{2}(r) g^{2}\left(\frac{1}{\boldsymbol{q}_{\perp}^{2}}-\frac{1}{\boldsymbol{q}_{\perp}^{2}+m_{\mathrm{D}}^{2}}\right)+y_{\ell}^{2} g^{\prime 2}\left(\frac{1}{\boldsymbol{q}_{\perp}^{2}}-\frac{1}{\boldsymbol{q}_{\perp}^{2}+m_{\mathrm{D}}^{\prime 2}}\right)\right] .
$$

Here $m_{\mathrm{D}}$ and $m_{\mathrm{D}}^{\prime}$ are the Debye masses of the $\mathrm{SU}(2)$ and $\mathrm{U}(1)$ gauge bosons. In the Standard Model they are given by [25]

$$
m_{\mathrm{D}}^{2}=\frac{11}{6} g^{2} T^{2}, \quad m_{\mathrm{D}}^{2}=\frac{11}{6} g^{\prime 2} T^{2} .
$$

With $C_{2}(r)$ we denote the quadratic Casimir operator which for the fundamental representation of SU(2) equals 3/4. Finally, the quantity $\epsilon(k, \boldsymbol{p})$, which gives the difference of the energy poles of Higgs and lepton propagator, reads

$$
\epsilon(k, \boldsymbol{p}) \equiv \frac{M_{N}^{2}}{2 k_{\|}}+\frac{\boldsymbol{p}_{\perp}^{2}+m_{\varphi}^{2}}{2\left(p_{\|}-k_{\|}\right)}-\frac{\boldsymbol{p}_{\perp}^{2}+m_{\ell}^{2}}{2 p_{\|}} .
$$

The equations (13) and (14) closely resemble those that are obtained for transverse and longitudinal photon production from a quark-gluon plasma [26, 27, 28] and can be solved numerically by using the same idea, namely to transform them via Fourier transformation into second-order ordinary differential equations with boundary values. Details and some numerical results can be found in [16]. 


\section{$4 \quad 2 \rightarrow 2$ scattering}

At leading order all external momenta in the $2 \rightarrow 2$ scattering processes are hard $(k \sim T)$. Furthermore, the typical angle between the initial state momenta or between the final state momenta is of order 1 . The masses of all external particles, including the mass of the right-handed neutrinos, are of order $g T$ and are neglected. The squared tree level matrix elements $|\mathscr{M}|^{2}$ only depend on the Mandelstam variables $s, t$, and $u$. When $|\mathscr{M}|^{2}$ is constant or proportional to $u / s$, the typical momentum transfer at leading order is hard. However when $|\mathscr{M}|^{2}$ is proportional to $s / t$ or $u / t$, there are leading order contributions from both hard and soft momentum transfer. A naive calculation using the tree level matrix elements would give an infrared divergent result. Therefore one has to introduce a scale that separates hard and soft momenta. When the momentum transfer is hard, one can use tree level matrix elements. For soft momentum transfer, on the other hand, one has to use the Hard Thermal Loop resummed propagator [29]. Both the hard and the soft contributions depend logarithmically on the separation scale, and only their sum is independent. 8

\subsection{Hard momentum transfer}

When all external momenta and the virtualities of internal momenta are hard, the LO production rate can simply be obtained from the Boltzmann equation [30]. More specifically, one can write the rate as

$$
\begin{aligned}
& (2 \pi)^{3} 2 k^{0} \frac{d \Gamma^{2 \rightarrow 2}}{d^{3} k}= \\
& \sum_{\text {processes }} \int\left(\prod_{a=1}^{3} \frac{d^{3} p_{a}}{(2 \pi)^{3} 2 E_{a}}\right)(2 \pi)^{4} \delta^{4}\left(p_{1}+p_{2}-p_{3}-k\right) f_{1} f_{2}\left(1 \pm f_{3}\right) \sum|\mathscr{M}|^{2},
\end{aligned}
$$

where $f_{a} \equiv f\left(E_{a}\right)$ are either Bose-Einstein or Fermi-Dirac distribution functions, and the upper/lower sign applies when particle 3 is a boson/fermion. Since the production rate is defined at negligible density of Majorana neutrinos, there are no disappearance processes and no Pauli blocking factor in Eq. (18). All momenta are hard and both the Majorana mass and the thermal masses are soft. Therefore all masses can be neglected and no propagator resummation is necessary. For the same reason we can put $E_{a}=\left|\boldsymbol{p}_{a}\right|$. The invariant matrix elements squared are listed in table 11. One also has to include the $C P$ conjugate processes, which is done by multiplying the rate by 2 .

\footnotetext{
${ }^{8}$ If the Majorana mass is not neglected, there are infrared divergences stemming from $t$-channel exchange of soft Higgs bosons [18. In our calculation these divergences are absent since we neglect the Majorana mass, consistent with our power counting $M_{N} \sim g T \ll T$.
} 


\begin{tabular}{|l|l|l|}
\hline Diagrams & Processes & $\sum|\mathscr{M}|^{2}$ \\
\hline$(a),(b),(c)$ & $Q_{3} \bar{t} \rightarrow \ell N, \bar{t} \bar{\ell} \rightarrow \bar{Q}_{3} N, Q_{3} \bar{\ell} \rightarrow t N$ & $6 h^{2} h_{t}^{2}$ \\
\hline$(d)$ & $\ell \varphi \rightarrow V N$ & $h^{2}\left(3 g^{2}+g^{\prime 2}\right) s /(-t)$ \\
\hline$(e)$ & $V \ell \rightarrow \bar{\varphi} N$ & $h^{2}\left(3 g^{2}+g^{\prime 2}\right)(-u) / s$ \\
\hline$(f)$ & $V \varphi \rightarrow \bar{\ell} N$ & $h^{2}\left(3 g^{2}+g^{\prime 2}\right) u / t$ \\
\hline
\end{tabular}

Table 1: Invariant matrix elements squared and summed over spins, colors, and weak isospin of the initial and final states, for the $2 \rightarrow 2$ scattering processes shown in Fig. 2 , The results for the charge conjugate processes are the same, and they are taken into account by multiplying the complete $2 \rightarrow 2$ rate by a factor of 2 .

In order to make the phase space integration as simple as possible, it is important to choose appropriate integration variables. We choose them such that the denominator in $\sum|\mathscr{M}|^{2}$ has no angular dependence, making the angular integrations straightforward. All but two integrations are performed analytically while the two remaining integrals are done numerically. When $\sum|\mathscr{M}|^{2}$ is constant or proportional to $u / s$ we define $q=p_{1}+p_{2}$. Then $s=q^{2}$ does not depend on any angle and many of the integrals in (18) can be trivially performed. On the other hand, when $\sum|\mathscr{M}|^{2}$ is proportional to $s / t$, we define $q=p_{1}-p_{3}$. In both cases we use the variables

$$
q_{+} \equiv \frac{1}{2}\left(q^{0}+|\boldsymbol{q}|\right), \quad q_{-} \equiv \frac{1}{2}\left(q^{0}-|\boldsymbol{q}|\right),
$$

together with the energy of one of the colliding particles for our non-trivial integrations. Further details can be found in Appendix A.

For processes $(d)$ and $(f)$ which involve a fermion exchange in the $t$-channel the full HTL resummed propagator needs to be used as soon as the momentum transfer becomes soft, while for a hard momentum a bare propagator is sufficient. A successful implementation was first given by Braaten and Yuan [31] and relies on introducing a cut for the square of the spatial momentum transfer. Here we proceed slightly differently from [31, 32, 33, 34, 35]. We introduce a cut for the square of the transverse momentum $\boldsymbol{q}_{\perp}$. By Eq. (A.17) $\boldsymbol{q}_{\perp}^{2}$ is determined by $q_{+}$and $q_{-}$. We take

$$
g T \ll q_{\text {cut }} \ll T,
$$

and integrate the tree level $2 \rightarrow 2$ scattering matrix elements only over transverse momenta with $\boldsymbol{q}_{\perp}^{2}>q_{\text {cut }}^{2}$. Due to (20) and (A.17) this condition turns out to be equivalent to $-q^{2}>q_{\text {cut }}^{2}$. The region $\boldsymbol{q}_{\perp}^{2}<q_{\text {cut }}^{2}$ is discussed in Sec. 4.2, There we will 
see why it is convenient to have a cut in transverse momenta only: Then we can use the sum rule for the HTL resummed propagator obtained in Appendix B.

We first perform all integrations except over $q_{+}$and $q_{-}$, see Eq. (A.15). The resulting integrand diverges like $1 / \boldsymbol{q}^{2}$ for $q \rightarrow 0$,

$$
\left[\frac{d \Gamma}{d^{3} k}\right]_{\text {hard }}=\int_{0}^{k^{0}} d q_{+} \int_{-\infty}^{0} d q_{-} \frac{F\left(q_{+}, q_{-}\right)}{\boldsymbol{q}^{2}} \Theta\left(\boldsymbol{q}_{\perp}^{2}-q_{\text {cut }}^{2}\right),
$$

which can be seen from Eqs. (A.15) and (A.16). Now we subtract and add the $q_{+}$, $q_{-} \rightarrow 0$ limit of $F$. This way we decompose (21) into a piece which is finite when $q_{\text {cut }} \rightarrow 0$, and one which is singular in this limit,

$$
\left[\frac{d \Gamma}{d^{3} k}\right]_{\text {hard }}=\left[\frac{d \Gamma}{d^{3} k}\right]_{\text {hard,finite }}+\left[\frac{d \Gamma}{d^{3} k}\right]_{\text {hard,singular }} .
$$

In the first term on the RHS we can put $q_{\text {cut }}=0$ which gives

$$
\left[\frac{d \Gamma}{d^{3} k}\right]_{\text {hard,finite }}=\int_{0}^{k^{0}} d q_{+} \int_{-\infty}^{0} d q_{-} \frac{F\left(q_{+}, q_{-}\right)-F(0,0)}{\boldsymbol{q}^{2}} .
$$

$F$ is obtained by combining Eqs. (18), (A.15) together with Eqs. (A.20) and (A.24). The remaining integrals have to be done numerically. The subtracted term is singular for $q_{\text {cut }} \rightarrow 0$, but it can be computed analytically. We find equal contributions for the processes $(d)$ and $(f)$. We also have to include the charge conjugated processes, which gives an additional factor 2 . The complete singular hard contribution is

$$
2 k^{0}(2 \pi)^{3}\left[\frac{d \Gamma}{d^{3} k}\right]_{\text {hard,singular }}=\frac{h^{2}}{2 \pi} m_{\ell}^{2} f_{\mathrm{B}}\left(k^{0}\right) \ln \left(\frac{2 k^{0}}{q_{\text {cut }}}\right)
$$

where $m_{\ell}$ is the asymptotic mass of the leptons (11).

\subsection{Soft momentum transfer}

Next we compute the contribution with soft transverse momentum transfer, $\boldsymbol{q}_{\perp}^{2}<q_{\text {cut }}$. Here we make use of (8) to compute the rate. The leading order contribution is given by the diagram in Fig. 4, where the internal lepton line is soft. The leading order selfenergy for soft fermions is given by a 1-loop diagram with a gauge field propagator and a hard loop momentum, a so-called Hard Thermal Loop (HTL). It is of the same order as the lepton momentum. Therefore one has to use the HTL resummed propagator as indicated by the thick blob in Fig. 4. The soft contribution to the rate depends on the separation scale $q_{\text {cut }}$, and adding the soft contribution and the hard singular gauge boson scattering rate (24) the separation scale drops out. 


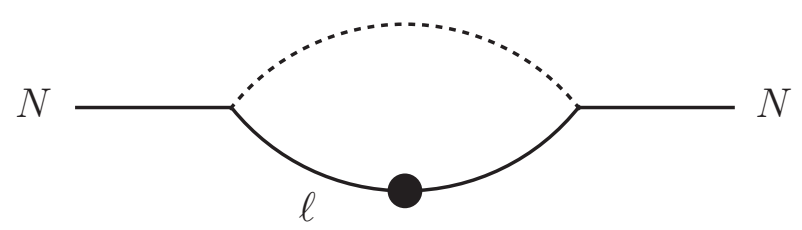

Figure 4: One-loop self-energy diagram of the Majorana neutrino. The black dot indicates that the lepton propagator is HTL resummed.

There is a similar contribution to thermal photon production which has been computed in Refs. [36, 37]. There it turned out that the result, which was found numerically, could be written in a simple analytic form [33, 36], despite the fact that the HTL selfenergy is a rather non-trivial function. Here we trace the reason why the complicated integrals in [33, 36, 37] have such a simple result back to a sum rule which is satisfied by the HTL resummed fermion propagator.

We must compute the imaginary part of the self-energy from Fig. 4 with a HTL resummed lepton propagator $S$ which is given by Eq. (B.1). The Higgs momentum is hard, so one can use the tree level Higgs propagator. In the imaginary time formalism the self-energy reads

$$
P_{\mathrm{L}} \Sigma(k)=d(r) h^{2} P_{\mathrm{L}} T \sum_{q^{0}} \int \frac{d^{3} q}{(2 \pi)^{3}} \Delta(k-q) S(q)
$$

with the free Higgs propagator $\Delta(p)=-1 / p^{2}$. After performing the sum over fermionic Matsubara frequencies $q^{0}$ one can analytically continue $k^{0}$ to real values.

One can decompose the fermion propagator according to $S=S^{\mu} \gamma_{\mu}$. In the calculation of the rate only the discontinuity

$$
\operatorname{disc} S(q) \equiv S\left(q^{0}+i 0^{+}, \boldsymbol{q}\right)-S\left(q^{0}+i 0^{-}, \boldsymbol{q}\right)
$$

across the real $q^{0}$-axis (with real $\boldsymbol{q}$ ) of the + component $S_{+} \equiv S_{0}+S_{z}=S^{0}-S^{z}$ enters. The integration over $q^{z}$ is done using the sum rule (see Appendix B)

$$
\int \frac{d q^{z}}{2 \pi} \operatorname{disc} S_{+}\left(q^{0}=q^{z}, \boldsymbol{q}_{\perp}\right)=\frac{i}{2} \frac{m_{\ell}^{2}}{\boldsymbol{q}_{\perp}^{2}+m_{\ell}^{2}} .
$$

Finally one can integrate over the transverse momenta with $\boldsymbol{q}_{\perp}^{2}<q_{\text {cut }}^{2}$ which gives

$$
2 k^{0}(2 \pi)^{3}\left[\frac{d \Gamma}{d^{3} k}\right]_{\mathrm{soft}}=\frac{h^{2}}{2 \pi} m_{\ell}^{2} f_{\mathrm{B}}\left(k^{0}\right) \ln \left(\frac{q_{\mathrm{cut}}}{m_{\ell}}\right) .
$$


Adding the singular hard contribution (24) and Eq. (28) the separation scale $q_{\text {cut }}$ drops out.

\section{$5 \quad$ Numerical results}

The total rate of the $2 \rightarrow 2$ processes can be written as

$$
\Gamma^{2 \rightarrow 2}=\frac{h^{2} T^{4}}{1536 \pi}\left\{h_{t}^{2} c_{Q}+\left(3 g^{2}+g^{\prime 2}\right)\left[\ln \left(\frac{1}{3 g^{2}+g^{\prime 2}}\right)+c_{V}\right]\right\},
$$

where $h_{t}$ is the top Yukawa coupling. We determined the constants $c_{Q}$ and $c_{V}$ numerically, with the results

$$
\begin{aligned}
& c_{Q}=2.52, \\
& c_{V}=3.17 .
\end{aligned}
$$

For the $1 \leftrightarrow 2$ processes there is no such simple expression, since the rate is a complicated function of the ratios of thermal and Debye masses.

To obtain numerical results for the rate we have to specify several parameters. The mass $M_{N}$ of the Majorana neutrino and its Yukawa couplings are unconstrained by lowenergy neutrino physics. If not stated otherwise we have chosen the exemplary value $M_{N}=10^{7} \mathrm{GeV}$ and we always plot our rates divided by $h^{2}$. The SM couplings are evaluated at the scale $\mu=2 \pi T$ using the 1-loop renormalization group equations [38]. The Higgs self-coupling $\lambda$ is determined by the zero temperature Higgs mass $m_{H}$ for which we have considered the two values 125 and $150 \mathrm{GeV}$.

In Fig. 5 we show rates for the various processes as a function $z \equiv M_{N} / T$. The $1 \leftrightarrow 2$ processes show the characteristic behavior found in [16]. Without gauge interaction (dotted and dot-dot-dashed lines) there is the Higgs decay at very high temperature and the inverse decay at lower temperature, and there is a gap in between where no process is kinematically allowed. Adding soft gauge interactions closes this gap. Even when the Higgs decay is allowed, gauge interactions enhance the rate by almost a factor 3. When the inverse $N$ decay is kinematically allowed, which happens close to $z=1$, the effect of gauge interactions becomes small, and our result for the complete $1 \leftrightarrow 2$ rate is approximately equal to the inverse decay rate of the right-handed neutrinos. This indicates that our $1 \leftrightarrow 2$ result which assumed the Majorana neutrino mass to be soft $\left(M_{N} \sim g T\right)$ would smoothly match onto the result for hard $\left(M_{N} \sim T\right)$ Majorana mass, for which the soft gauge interactions are suppressed. However, we cannot expect our $2 \rightarrow 2$ results to be valid in this region since they were obtained by neglecting $M_{N}$. 


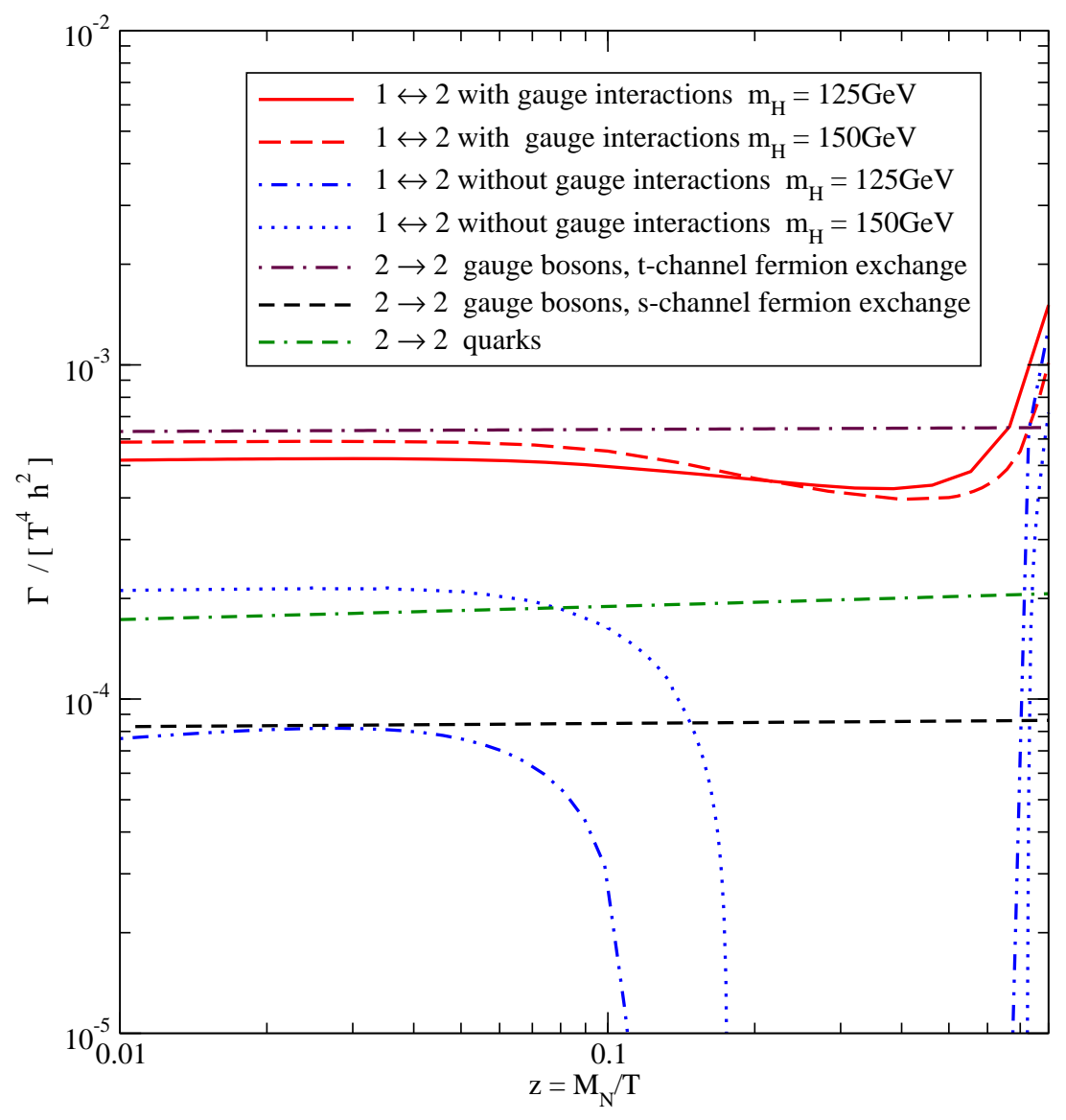

Figure 5: Dependence of the production rate on $z$ for $M_{N}=10^{7} \mathrm{GeV}$.

The contribution from $2 \rightarrow 2$ gauge boson scattering with $t$-channel fermion exchange is about the same size as from the $1 \leftrightarrow 2$ scattering with soft gauge interaction. This shows that gauge bosons, both real and virtual, are essential for the production, ignoring them would strongly underestimate the rate. The quark scattering contributions are smaller than the gauge boson scattering by more than a factor 3, despite the large top Yukawa coupling. The rates of the $2 \rightarrow 2$ processes depend only logarithmically on $z$ through the running of the gauge coupling, since we have neglected the Majorana mass in the $2 \rightarrow 2$ matrix elements

Refs. [19, 20] find that the $1 \leftrightarrow 2$ processes without soft gauge interaction give a much smaller rate at high $T$ than the $2 \rightarrow 2$ scattering. This is because they do not include thermal masses in the $1 \leftrightarrow 2$ rates which are, however, crucial at high temperature. Thermal masses were included in Ref. [14]. The resulting rate is about $60 \%$ larger than ours. This could be related to the fact that [14 uses the mass for soft fermion excitations instead of the asymptotic lepton mass $m_{\ell}$ which is larger by a 


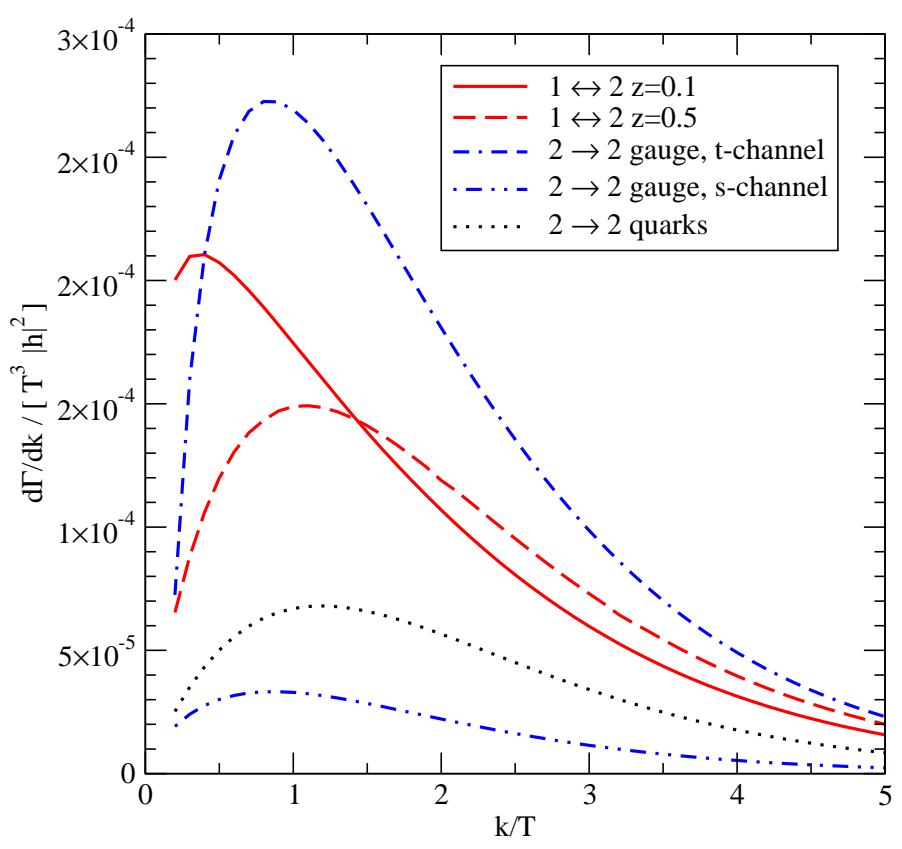

Figure 6: Momentum spectrum of the produced right-handed neutrinos for $M_{N}=10^{7}$ $\mathrm{GeV}$ and $m_{H}=125 \mathrm{GeV} . s^{-}$and $t$-channel refers to the exchanged fermion.

factor $\sqrt{2}$, thus overestimating the rate for Higgs decay. In Ref. [14] it was found that at very high $T$ the complete $2 \rightarrow 2$ scattering rate is approximately equal to the rate due to Higgs decay without soft gauge interaction (see Eq. (4) ). 9 Here we find that the former gives a much larger contribution. This discrepancy cannot only be due to the use of the soft fermion mass in Ref. [14]. Another source of discrepancy could be that in [14, 19] the soft fermion exchange is dealt with differently from our complete leading order treatement. Both [19] and [14] find that at high temperatures the $2 \rightarrow 2$ gauge boson scattering dominates over the quark scattering by about a factor 2 . Here we find that the gauge boson scattering dominates by a much larger factor of about 5 .

Fig. [6 shows the momentum spectrum of the produced neutrinos. For $2 \rightarrow 2$ processes the typical momentum is near $T$. This is also the case for the $1 \leftrightarrow 2$ contribution at $z=0.5$, where without gauge interaction no decay or inverse decay is allowed. However in the high temperature regime where the Higgs decay is allowed the spectrum is much more infrared. This does not invalidate our calculation, because it is still applicable when the right-handed neutrinos are soft.

In Fig. 7 we show the temperature dependence of the production rate for vanishing

\footnotetext{
${ }^{9}$ If one wants to compare the individual contributions of [14 with ours one has to be careful: In the present paper $s$ - or $t$-channel refers to the fermion exchange, while in Ref. 14] it refers to the Higgs exchange.
} 


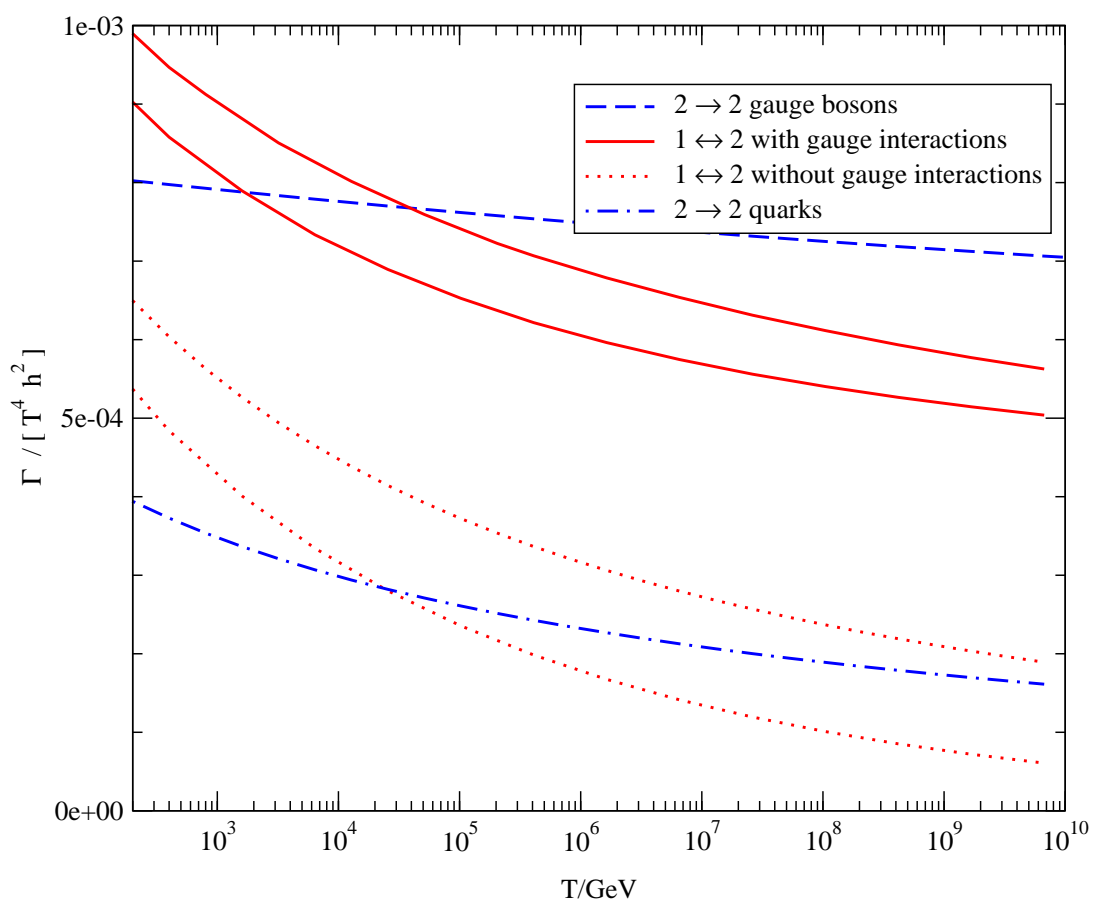

Figure 7: Production rate of massless right-handed neutrinos as a function of the temperature. The upper and lower $1 \leftrightarrow 2$ curves are for $m_{H}=150$ and $125 \mathrm{GeV}$, respectively.

Majorana mass. This is of particular importance for leptogenesis which occurs far from thermal equilibrium at relatively low temperature, for instance below the electroweak phase transition [12]. For all temperatures between 100 and $10^{10} \mathrm{GeV}$ the rates for $1 \leftrightarrow 2$ scattering plus soft gauge interaction and the gauge boson scattering are of similar size, and they dominate over the quark scattering. At lower temperatures the $1 \leftrightarrow 2$ scattering gives the largest contribution, while at higher $T$ the $2 \rightarrow 2$ gauge boson scattering takes over. Numerical results for the $1 \leftrightarrow 2$ production rate are also shown in Tab. 2.

Another interesting result of this paper is the Higgs mass dependence of the $1 \leftrightarrow 2$ rate which is displayed in Figs. 5 and 7 . Without gauge interactions we observe a strong suppression in the region where the Higgs can decay, while the rate gets enhanced in the region of inverse $N$-decay. When gauge interactions are included, the relative $m_{H}$-dependence is much weaker. 


\begin{tabular}{c|c}
$T / \mathrm{GeV}$ & $\Gamma /\left(h^{2} T^{4}\right)$ \\
\hline $1.00 \times 10^{2}$ & $9.5 \times 10^{-4}$ \\
$1.60 \times 10^{3}$ & $7.9 \times 10^{-4}$ \\
$2.56 \times 10^{4}$ & $6.9 \times 10^{-4}$ \\
$4.10 \times 10^{5}$ & $6.2 \times 10^{-4}$ \\
$6.55 \times 10^{6}$ & $5.7 \times 10^{-4}$ \\
$1.05 \times 10^{8}$ & $5.4 \times 10^{-4}$ \\
$1.68 \times 10^{9}$ & $5.1 \times 10^{-4}$ \\
$6.71 \times 10^{9}$ & $5.0 \times 10^{-4}$
\end{tabular}

Table 2: Production rate $\Gamma$ of massless right-handed neutrinos from $1 \leftrightarrow 2$ scattering including gauge interactions for $m_{H}=125 \mathrm{GeV}$.

\section{Summary and Conclusions}

In this paper we have computed the complete leading order production rate of righthanded neutrinos at high temperature, assuming that their mass $M_{N}$ is soft, $M_{N} \sim g T$. There are two types of contributions. The first is due to $1 \leftrightarrow 2$ scattering processes with additional soft gauge interactions. They are characterized by the near collinearity of the participating hard particles, and we have computed them before. Here we have studied their dependence on the zero temperature Higgs mass, which turned out to be weak when soft gauge interactions are included. We have completed the leading order calculation by also including $2 \rightarrow 2$ scattering processes. The $2 \rightarrow 2$ processes involving electroweak gauge bosons were consistently computed for the first time by properly treating the effects of the hot plasma on soft lepton exchange.

The gauge boson $2 \rightarrow 2$ rate is of similar size as the complete $1 \leftrightarrow 2$ rate. Both are significantly larger than the top quark scattering contribution despite the large top Yukawa coupling. At lower temperatures the $1 \leftrightarrow 2$ processes give the largest contribution, while at higher temperature the $2 \rightarrow 2$ rate takes over. Our main conclusion is thus that processes involving gauge interactions are the most important ones for producing right-handed neutrinos at high temperature. At higher order the processes discussed in this paper also contribute to the CP asymmetry. Thus one should expect gauge interactions to play a crucial role also in that context where they have not been taken into account so far [14, 39].

Acknowledgments DB thanks Björn Garbrecht and Mikko Laine for useful discussions and suggestions, and Mikko Laine for pointing out an error in an earlier version of this paper. This work was supported in part through the DFG funded Graduate School GRK 881. 


\section{A Details of the calculation of the $2 \rightarrow 2$ rate}

The phase space integrations in Eq. (18) are done as follows. Following [32, 33] we introduce the 4-momentum $q$ of an exchanged virtual particle such that $q^{2}$ appears in the denominator of the squared matrix elements. After doing some angular integrals we end up with non-trivial integrations over $q_{+}, q_{-}$, which are defined in Eq. (19), and the energy $E^{\prime}$ of one of the colliding particles. For each process the products of Bose and Fermi distribution functions in Eq. (18) are written in the form

$$
f_{1} f_{2}\left[1 \pm f_{3}\right]=f_{\mathrm{F}}\left(k^{0}\right) \tilde{f} \widehat{f}
$$

where $k^{0}$ is the energy of the produced right-handed neutrino. $\tilde{f}$ and $\widehat{f}$ are both linear combinations of Bose and Fermi distribution functions which are process dependent. They are chosen such that only $\widehat{f}$ depends on $E^{\prime}$. This greatly simplifies the integrals over $E^{\prime}$ which are then performed analytically. In order to arrive at Eq. (A.1) we use the identity

$$
f_{\mathrm{B}}\left(E_{1}\right) f_{\mathrm{B}}\left(E_{2}\right)=f_{\mathrm{B}}\left(E_{1}+E_{2}\right)\left[1+f_{\mathrm{B}}\left(E_{1}\right)+f_{\mathrm{B}}\left(E_{2}\right)\right]
$$

and the corresponding relations for Fermi distributions which are obtained using

$$
f_{\mathrm{F}}(\omega)=-f_{\mathrm{B}}(\omega+i \pi T),
$$

together with

$$
f_{\mathrm{B}}(-\omega)=-\left[1+f_{\mathrm{B}}(\omega)\right]
$$

The remaining integrations over $q_{+}$and $q_{-}$are done numerically. In the processes with fermion exchange in the $t$-channel we perform a subtraction in the integrand to isolate the singular contribution.

\section{A.1 s-channel parametrization}

For gauge boson scattering with $s$-channel fermion exchange, where $\sum|\mathscr{M}|^{2}$ is proportional to $u / s$, and for quark scattering where it is constant, we choose

$$
q=p_{3}+k
$$

so that $s=q^{2}$, and $E^{\prime}=E_{2}$. For $n=0$ or $n=1$ we obtain

$$
\begin{aligned}
\int\left[\prod_{a=1}^{3} \frac{d^{3} p_{a}}{(2 \pi)^{3} 2 E_{a}}\right] & (2 \pi)^{4} \delta\left(p_{1}+p_{2}-p_{3}-k\right) \tilde{f} \widehat{f}\left(\frac{-u}{s}\right)^{n} \\
& =\frac{1}{32 \pi^{3} k^{0}} \int_{k^{0}}^{\infty} d q_{+} \int_{0}^{k^{0}} d q_{-} \tilde{f} \int_{q_{-}}^{q_{+}} d E_{2} \widehat{f}\left(\frac{\langle-u\rangle}{s}\right)^{n}
\end{aligned}
$$


with

$$
\frac{\langle-u\rangle}{s}=\frac{1}{\boldsymbol{q}^{2}}\left(\frac{1}{2}\left(q_{0}^{2}+\boldsymbol{q}^{2}\right)-q^{0}\left(E_{2}+k^{0}\right)+2 E_{2} k^{0}\right)
$$

and $q^{0}=q_{+}+q_{-}, q^{2}=4 q_{+} q_{-}$, and $|\boldsymbol{q}|=q_{+}-q_{-}$. The angular brackets indicate that the Mandelstam variable $t$ has been averaged over the azimuth of $\boldsymbol{p}_{2}$ with respect to $\boldsymbol{q}$. We have to distinguish two cases:

\section{A.1.1 quark scattering}

All external particles are fermions, and we use

$$
\begin{aligned}
& \widetilde{f}=f_{\mathrm{B}}\left(E_{3}+k^{0}\right)+f_{\mathrm{F}}\left(E_{3}\right), \\
& \widehat{f}=1-f_{\mathrm{F}}\left(E_{1}\right)-f_{\mathrm{F}}\left(E_{2}\right),
\end{aligned}
$$

where $E_{3}=q^{0}-k^{0}$ and $E_{1}=q^{0}-E_{2} . \sum|\mathscr{M}|^{2}$ is constant which corresponds to $n=0$ in Eq. (A.6) and we find

$$
\int_{q_{-}}^{q_{+}} d E_{2} \widehat{f}=|\boldsymbol{q}|+2 T\left[\log \left(1+e^{-q_{+} / T}\right)-\log \left(1+e^{-q_{-} / T}\right)\right] .
$$

\section{A.1.2 $V \ell \rightarrow \bar{\varphi} N$}

Here we have both external fermions and bosons, so that

$$
\begin{aligned}
& \widetilde{f}=f_{\mathrm{F}}\left(E_{3}+k^{0}\right)+f_{\mathrm{B}}\left(E_{3}\right), \\
& \widehat{f}=1+f_{\mathrm{B}}\left(E_{1}\right)-f_{\mathrm{F}}\left(E_{2}\right) .
\end{aligned}
$$

The $E_{2}$-integral in Eq. (A.6) gives

$$
\begin{gathered}
\int_{q_{-}}^{q_{+}} d E_{2} \widehat{f} \frac{\langle-u\rangle}{s}=\frac{|\boldsymbol{q}|}{2}-\frac{T}{|\boldsymbol{q}|}\left[\left(k^{0}-q_{+}\right)\left(\ln \left(1-e^{-q_{+} / T}\right)-\ln \left(1+e^{-q_{-} / T}\right)\right)\right. \\
\left.\quad+\left(k^{0}-q_{-}\right)\left(\ln \left(1-e^{-q_{-} / T}\right)-\ln \left(1+e^{-q_{+} / T}\right)\right)\right] \\
-\frac{T^{2}}{\boldsymbol{q}^{2}}\left(q^{0}-2 k^{0}\right)\left[\operatorname{Li}_{2}\left(e^{-q_{+} / T}\right)-\operatorname{Li}_{2}\left(e^{-q_{-} / T}\right)-\mathrm{Li}_{2}\left(-e^{-q_{+} / T}\right)+\operatorname{Li}_{2}\left(-e^{-q_{-} / T}\right)\right],
\end{gathered}
$$

where $\mathrm{Li}_{2}$ is the dilogarithm.

\section{A.2 $t$-channel parametrization: hard contribution}

For a process with a fermion exchange in the $t$-channel we choose

$$
q=p_{1}-p_{3},
$$


so that $t=q^{2}$, and $E^{\prime}=E_{1}$. For the phase space integral in Eq. (18) we obtain

$$
\begin{aligned}
\int\left[\prod_{a=1}^{3} \frac{d^{3} p_{a}}{(2 \pi)^{3} 2 E_{a}}\right] & (2 \pi)^{4} \delta\left(p_{1}+p_{2}-p_{3}-k\right) \tilde{f} \widehat{f}\left(\frac{s}{-t}\right)^{n} \\
& =\frac{1}{32 \pi^{3} k^{0}} \int_{0}^{k^{0}} d q_{+} \int_{-\infty}^{0} d q_{-} \tilde{f} \int_{q_{+}}^{\infty} d E_{1} \widehat{f}\left(\frac{\langle s\rangle}{-t}\right)^{n}
\end{aligned}
$$

where we need the cases $n=0$ and $n=1$. Furthermore,

$$
\frac{\langle s\rangle}{-t}=\left(\boldsymbol{q}^{2}+2 E_{1} k^{0}-q^{0}\left(E_{1}+k^{0}\right)+\frac{q^{2}}{2}\right) / \boldsymbol{q}^{2}
$$

with $q^{0}, q^{2}$, and $\boldsymbol{q}^{2}$ as in Eq. (A.6). The transverse momentum transfer is given by

$$
\boldsymbol{q}_{\perp}^{2}=-\frac{q^{2}}{k_{0}^{2}}\left(k_{0}-q_{+}\right)\left(k_{0}-q_{-}\right) .
$$

Thus for small $q$ we have $\boldsymbol{q}_{\perp}^{2} \simeq-q^{2}$. Now the angular brackets indicate an average over the azimuth of $\boldsymbol{p}_{1}$ with respect to $\boldsymbol{q}$. During $E_{1}$-integration $E_{3}=E_{1}-q^{0}$ changes, while $E_{2}=k^{0}-q^{0}$ remains fixed. Again we have to distinguish two cases:

\section{A.2.1 $\ell \varphi \rightarrow V N$}

In this case

$$
\begin{aligned}
& \tilde{f}=1+f_{\mathrm{B}}\left(E_{2}\right)-f_{\mathrm{F}}\left(q^{0}\right), \\
& \widehat{f}=f_{\mathrm{F}}\left(E_{1}\right)+f_{\mathrm{B}}\left(E_{3}\right) .
\end{aligned}
$$

The matrix element squared is proportional to $s / t$, so that we have the $E_{1}$-integral

$$
\begin{aligned}
\int_{q_{+}}^{\infty} d E_{1} \hat{f} \frac{\langle s\rangle}{-t}= & \frac{T}{|\boldsymbol{q}|}\left(k^{0}-q_{-}\right)\left[\ln \left(1+e^{-q_{+} / T}\right)-\ln \left(1-e^{q_{-} / T}\right)\right] \\
& +\frac{T^{2}}{\boldsymbol{q}^{2}}\left(2 k^{0}-q^{0}\right)\left[-\operatorname{Li}_{2}\left(-e^{-q_{+} / T}\right)+\operatorname{Li}_{2}\left(e^{q_{-} / T}\right)\right] .
\end{aligned}
$$

For the subtraction in Eq. (23) we need the small $q$ limit of (A.20) for which we find

$$
\int_{q_{+}}^{\infty} d E_{1} \hat{f} \frac{\langle s\rangle}{-t}=\frac{\pi^{2} T^{2} k^{0}}{2 \boldsymbol{q}^{2}}+O\left(q^{-1}\right) .
$$




\section{A.2.2 $V \varphi \rightarrow \bar{\ell} N$}

Now we have

$$
\begin{aligned}
& \widetilde{f}=1+f_{\mathrm{B}}\left(E_{2}\right)-f_{\mathrm{F}}\left(q^{0}\right), \\
& \widehat{f}=f_{\mathrm{B}}\left(E_{1}\right)+f_{\mathrm{F}}\left(E_{3}\right) .
\end{aligned}
$$

The matrix element squared is proportional to $u / t=-1-s / t$, and the integration over $E_{1}$ yields

$$
\begin{aligned}
\int_{q_{+}}^{\infty} d E_{1} \hat{f} \frac{\hat{u}\rangle}{t}= & \frac{T}{|\boldsymbol{q}|}\left(k^{0}-q_{+}\right)\left[-\ln \left(1-e^{-q_{+} / T}\right)+\ln \left(1+e^{q_{-} / T}\right)\right] \\
& +\frac{T^{2}}{\boldsymbol{q}^{2}}\left(2 k^{0}-q^{0}\right)\left[\operatorname{Li}_{2}\left(e^{-q_{+} / T}\right)-\operatorname{Li}_{2}\left(-e^{q_{-} / T}\right)\right] .
\end{aligned}
$$

The term which is subtracted in Eq. (23) is the same as for the processes $\ell \varphi \rightarrow V N$ because

$$
\int_{q_{+}}^{\infty} d E_{1} \hat{f} \frac{\langle u\rangle}{t}=\frac{\pi^{2} T^{2} k^{0}}{2 \boldsymbol{q}^{2}}+O\left(q^{-1}\right)
$$

is the same as (A.21).

\section{B Sum rule for HTL resummed fermion propagotor}

Here we show that the Hard Thermal Loop (HTL) resummed propagator for massless fermions

$$
S(q)=\frac{-1}{q-\Sigma_{\mathrm{HTL}}(q)}
$$

satisfies the sum rule (27). $\Sigma_{\mathrm{HTL}}=\Sigma_{\mathrm{HTL}}^{\mu} \gamma_{\mu}$ is the HTL self-energy [22, 40]. Its components can be written as

$$
\begin{aligned}
& \Sigma_{\mathrm{HTL}}^{0}(q)=\frac{m_{\ell}^{2}}{4} q^{0} \int_{-1}^{1} \frac{d x}{\left(q^{0}\right)^{2}-x^{2} \boldsymbol{q}^{2}} \\
& \boldsymbol{\Sigma}_{\mathrm{HTL}}(q)=\frac{\boldsymbol{q}}{\boldsymbol{q}^{2}}\left(q^{0} \Sigma_{\mathrm{HTL}}^{0}-\frac{m_{\ell}^{2}}{2}\right)
\end{aligned}
$$

with the asymptotic thermal mass $m_{\ell}$ (see Eq. (11)). The sum rule (27) is similar to the one for the gauge field propagator found in Ref. [1]. The reason why the complicated HTL resummed propagator gives such a simple result has been identified in 42. Due to causality, the propagator is analytic not only in the upper and lower 
half of the complex $q^{0}$-plane, but also when an imaginary light-like vector is added to q. This also holds for the fermion propagator as can be easily seen from Eqs. (B.2) and (B.3). Therefore one can move the integration contour in Eq. (27) for the first term in Eq. (26) into the upper $q^{z}$-half plane and for the second one into the lower half plane. The integrand $S_{+}$falls off like $1 / q^{z}$ for $\left|q^{z}\right| \rightarrow \infty$. Closing the integration contour at infinity one then obtains (27).

\section{References}

[1] E. Komatsu et al. [WMAP Collaboration], Seven-Year Wilkinson Microwave Anisotropy Probe (WMAP) Observations: Cosmological Interpretation, Astrophys. J. Suppl. 192 (2011) 18 [arXiv:1001.4538 [astro-ph.CO]].

[2] A. D. Sakharov, Violation of CP invariance, C asymmetry, and baryon asymmetry of the Universe, Pisma Zh. Eksp. Teor. Fiz. 5 (1967) 32.

[3] For a review see e.g. W. Buchmüller, Baryogenesis-40 years after, [arXiv:0710.5857v2].

[4] M. Fukugita and T. Yanagida, Baryogenesis without grand unification, Phys. Lett. B 174 (1986) 45.

[5] V. Kuzmin, V. Rubakov and M. Shaposhnikov, On anomalous electroweak baryonnumber nonconservation in the early universe, Phys. Lett. B 155 (1985) 36.

[6] P. Minkowski, $\mu \rightarrow$ e $\gamma$ at a rate of one out of $10^{9}$ muon decays?, Phys. Lett. B 67 (1977) 421; M. Gell-Mann, P. Ramond and R. Slansky, Complex spinors and unified theories, in: Proc.Supergravity Stony Brook Workshop, New York 1979, ed. by P. van Nieuwenhuizen and D.Z. Freedman, North-Holland Publ. Co., Amsterdam, 1979 ; T. Yanagida, Horizontal symmetries and masses of neutrinos, Prog. Theor. Phys. 64 (1980), 1103.

[7] A. Anisimov, W. Buchmüller, M. Drewes and S. Mendizabal, Leptogenesis from Quantum Interference in a Thermal Bath, Phys. Rev. Lett. 104 (2010) 121102 [arXiv:1001.3856 [hep-ph]].

[8] M. Shaposhnikov, The nuMSM, leptonic asymmetries, and properties of singlet fermions, JHEP 0808 (2008) 008 [arXiv:0804.4542 [hep-ph]]. 
[9] M. Laine and Y. Schröder, Thermal right-handed neutrino production rate in the non-relativistic regime, arXiv:1112.1205 [hep-ph].

[10] A. Salvio, P. Lodone and A. Strumia, Towards leptogenesis at NLO: the righthanded neutrino interaction rate, JHEP 1108 (2011) 116 [arXiv:1106.2814 [hepph]].

[11] E. K. Akhmedov, V. A. Rubakov and A. Y. Smirnov, Baryogenesis via neutrino oscillations, Phys. Rev. Lett. 81 (1998) 1359 [arXiv:hep-ph/9803255].

[12] T. Asaka and M. Shaposhnikov, The nuMSM, dark matter and baryon asymmetry of the universe, Phys. Lett. B 620 (2005) 17 [arXiv:hep-ph/0505013].

[13] T. Asaka, S. Blanchet and M. Shaposhnikov, The nuMSM, dark matter and neutrino masses, Phys. Lett. B 631 (2005) 151 [arXiv:hep-ph/0503065].

[14] G. F. Giudice, A. Notari, M. Raidal, A. Riotto and A. Strumia, Towards a complete theory of thermal leptogenesis in the SM and MSSM, Nucl. Phys. B 685 (2004) 89 [arXiv:hep-ph/0310123].

[15] C.P. Kiessig, M. Plümacher and M.H. Thoma, Decay of a Yukawa fermion at finite temperature and applications to leptogenesis, Phys. Rev. D 82 (2010) 036007 [arXiv:1003.3016v2 [hep-ph]].

[16] A. Anisimov, D. Besak and D. Bödeker, Thermal production of relativistic Majorana neutrinos: Strong enhancement by multiple soft scattering, JCAP 1103 (2011) 042 [arXiv:1012.3784 [hep-ph]].

[17] A. Anisimov, W. Buchmüller, M. Drewes and S. Mendizabal, Quantum Leptogenesis I, Annals Phys. 326 (2011) 1998 [arXiv:1012.5821 [hep-ph]].

[18] M. A. Luty, Baryogenesis via leptogenesis, Phys. Rev. D 45 (1992) 455.

[19] A. Pilaftsis and T. Underwood, Resonant leptogenesis, Nucl. Phys. B 692 (2004) 303 [arXiv:hep-ph/0309342].

[20] F. Hahn-Woernle, M. Plümacher and Y. Wong, Full Boltzmann equations for leptogenesis including scattering, JCAP 0908 (2009) 028 [arXiv:0907.0205].

[21] W. Buchmüller, P. Di Bari and M. Plümacher, Cosmic microwave background, matter - antimatter asymmetry and neutrino masses, Nucl. Phys. B 643 (2002) 367 [Erratum-ibid. B 793 (2008) 362] [arXiv:hep-ph/0205349]. 
[22] H. A. Weldon, Effective fermion masses of order gT in high-temperature gauge theories with exact chiral invariance, Phys. Rev. D 26 (1982) 2789.

[23] V. V. Lebedev and A. V. Smilga, Supersymmetric sound, Nucl. Phys. B 318 (1989) 669.

[24] S. Caron-Huot, On supersymmetry at finite temperature, Phys. Rev. D 79 (2009) 125002 [arXiv:0808.0155 [hep-th]].

[25] M. E. Carrington, The Effective potential at finite temperature in the Standard Model, Phys. Rev. D 45 (1992) 2933.

[26] P. Arnold, G. D. Moore and L. G. Yaffe, Photon Emission from Ultrarelativistic Plasmas, JHEP 0111 (2001) 057 [arXiv:hep-ph/0109064].

[27] D. Besak and D. Bödeker, Hard Thermal Loops for soft or collinear external momenta, JHEP 1005 (2010) 007 [arXiv:1002.0022 [hep-ph]].

[28] P. Aurenche, F. Gelis, G.D. Moore and H. Zaraket, Landau-PomeranchukMigdal resummation for dilepton production, JHEP 0212 (2002) 006 [arXiv:hep$\mathrm{ph} / 0211036]$.

[29] E. Braaten and R. D. Pisarski, Soft Amplitudes in Hot Gauge Theories: A General Analysis, Nucl. Phys. B 337 (1990) 569; J. Frenkel and J. C. Taylor, High Temperature Limit of Thermal QCD, Nucl. Phys. B 334 (1990) 199.

[30] H. A. Weldon, Simple rules for discontinuities in finite-temperature field theory, Phys. Rev. D 28 (1983) 2007.

[31] E. Braaten and T. C. Yuan, Calculation of screening in a hot plasma, Phys. Rev. Lett. 66 (1991) 2183.

[32] G. D. Moore, Transport coefficients in large N(f) gauge theory: Testing hard thermal loops, JHEP 0105 (2001) 039 [arXiv:hep-ph/0104121].

[33] P. Arnold, G. D. Moore and L. G. Yaffe, Photon emission from quark gluon plasma: Complete leading order results, JHEP 0112 (2001) 009 [arXiv:hep-ph/0111107].

[34] M. Bolz, A. Brandenburg and W. Buchmüller, Thermal production of gravitinos, Nucl. Phys. B 606 (2001) 518 [arXiv:hep-ph/0012052].

[35] A. Brandenburg and F.D. Steffen, Axino dark matter from thermal production, JCAP 0408 (2004) 008 [arXiv:hep-ph/0405158]. 
[36] J. I. Kapusta, P. Lichard and D. Seibert, High-energy photons from quark - gluon plasma versus hot hadronic gas, Phys. Rev. D 44 (1991) 2774 [Erratum-ibid. D 47 (1993) 4171]

[37] R. Baier, H. Nakkagawa, A. Niegawa and K. Redlich, Production rate of hard thermal photons and screening of quark mass singularity, Z. Phys. C 53 (1992) 433.

[38] H. Arason et al., Renormalization-group study of the standard model and its extensions: The standard model, Phys. Rev. D 46 (1992) 3945; B. Schrempp and M. Wimmer, Top quark and Higgs boson masses: Interplay between infrared and ultraviolet physics, Prog. Part. Nucl. Phys. 37, 1, 1996 [arXiv:hep-ph/9606386].

[39] L. Covi, N. Rius, E. Roulet and F. Vissani, Finite temperature effects on CP violating asymmetries, Phys. Rev. D 57 (1998) 93 [arXiv:hep-ph/9704366]; M. Garny, A. Hohenegger and A. Kartavtsev, Medium corrections to the CP-violating parameter in leptogenesis, Phys. Rev. D 81 (2010) 085028 [arXiv:1002.0331 [hep-ph]]; C. Kiessig and M. Plümacher, Hard-Thermal-Loop Corrections in Leptogenesis I: CP-Asymmetries, arXiv:1111.1231 [hep-ph].

[40] V. V. Klimov, Spectrum of Elementary Fermi Excitations in Quark Gluon Plasma (In Russian), Sov. J. Nucl. Phys. 33 (1981) 934 [Yad. Fiz. 33 (1981) 1734]; O. K. Kalashnikov, QCD at finite temperature, Fortsch. Phys. 32 (1984) 525.

[41] P. Aurenche, F. Gelis and H. Zaraket, A simple sum rule for the thermal gluon spectral function and applications, JHEP 0205 (2002) 043 [arXiv:hep-ph/0204146].

[42] S. Caron-Huot, O(g) plasma effects in jet quenching, Phys. Rev. D 79 (2009) 065039 [arXiv:0811.1603 [hep-ph]]. 